\title{
Immigration, Local Dispersal Limitation, and the Repeatability of Community Composition under Neutral and Niche Dynamics
}

\author{
Dexiecuo Ai ${ }^{1}$, Philippe Desjardins-Proul ${ }^{2,3,4}$, Chengjin $\mathrm{Chu}^{1}$, Gang Wang ${ }^{1 *}$ \\ 1 School of Life Sciences, Lanzhou University, Lanzhou, China, 2 Theoretical Ecosystem Ecology Laboratory, Université du Québec à Rimouski, Rimouski, Canada, 3 Quebec \\ Center for Biodiversity Science, McGill University, Montréal, Canada, 4 College of Engineering, University of Illinois at Chicago, Chicago, Illinois, United States of America
}

\begin{abstract}
Repeatability of community composition has been a critical aspect for community structure, which is closely associated with community stability, predictability, conservation biology and ecological restoration. It has been shown that both immigration and local dispersal limitation can affect the community composition in both neutral and niche model. Hence, we use a spatially explicit individual-based model to investigate the potential influence of immigration rate and strength of local dispersal limitation on repeatability in both neutral and niche models. Similarity measures are used to quantify repeatability. We examine the repeatability of community composition among replicate communities (which means the same community repeats many times), and between niche and neutral replicate communities. We find the correlation between repeatability and immigration rate is positive in the neutral model and an inverted unimodal in the niche model. The correlation between repeatability and local dispersal distance is positive in the niche model and negative in the neutral model. High repeatability between niche communities and neutral communities is observed with high immigration rates or when high local dispersal distance appears in the niche model or low local dispersal distance in the neutral model. Our results show that repeatability of community composition is not only dependent on the types of community models (niche vs. neutrality) but also strongly determined by immigration rates and local dispersal limitation.
\end{abstract}

Citation: Ai D, Desjardins-Proulx P, Chu C, Wang G (2012) Immigration, Local Dispersal Limitation, and the Repeatability of Community Composition under Neutral and Niche Dynamics. PLoS ONE 7(9): e46164. doi:10.1371/journal.pone.0046164

Editor: Nicolas Mouquet, CNRS, University of Montpellier II, France

Received March 25, 2012; Accepted August 30, 2012; Published September 24, 2012

Copyright: (c) $2012 \mathrm{Ai}$ et al. This is an open-access article distributed under the terms of the Creative Commons Attribution License, which permits unrestricted use, distribution, and reproduction in any medium, provided the original author and source are credited.

Funding: The study was supported by the National Natural Science Foundation of China (No. 30970465). The funders had no role in study design, data collection and analysis, decision to publish, or preparation of the manuscript.

Competing Interests: The authors have declared that no competing interests exist.

* E-mail: wgmg36@|zu.edu.cn

\section{Introduction}

Repeatability of community composition is a key property of community stability and predictability [1-5], which also plays a major role in conservation biology and ecological restoration and can be used as the foundation for regional conservation planning [6]. For example, in many instances it is desired that similar assemblages arise after large disturbances, e.g. in population management and conservation [7]. Here, we define the repeatability as the similarity of community composition among replicate communities which means that the same community repeats many times, assembled under identical conditions [7].

The repeatability of community composition is one of the important aspects of community assembly [7], like species diversity and relative species abundance distribution [8-11]. The factors influencing the community composition are different between classic niche and neutral theory. In classic niche theory, community composition is determined by species-specific difference and heterogeneous environment [11-14]. However, in neutral theory, it is determined by ecological drift and immigration rate $[8,15,16]$. In addition, more studies have showed that incorporating immigration and local dispersal limitation with niche theory can preferably explain community composition; meanwhile these two factors also play important roles in neutral theory [17-19].
It has well demonstrated that immigration is a potential factor affecting community similarity [20,21]. Without immigration, the niche theory is insufficient to explain the species richness observed in nature, because the number of coexisting species in classic niche communities will be no greater than the number of limiting resources [11,22-24]. However, high immigration rates will compensate for a low species persistence through mass- or rescue-effects [25-27]. Loreau and Mouquet [18] investigated the influence of immigration from a regional pool on a plant community governed by competition for space and found that immigration could have a huge effect on local species diversity in competitive communities. In neutral theory, species diversity is maintained by a stochastic balance between extinction and immigration $[8,9,17]$. In the absence of immigration and speciation, local neutral communities will collapse to a single species [28]. Thus, immigration is a critical component for both the niche and neutral models.

Closely associated with immigration, dispersal limitation is another important factor influencing the community composition [19,29]. In Hubbell's neutral model [8], the dispersal limitation is implemented by limiting the immigration rate from the outside and it occurs between local communities or between local community and metacommunity. Here, we define local dispersal limitation (hereafter simply referred to as dispersal limitation) as 
spatially limited dispersal in local communities [19,29,30]. Dispersal limitations determine patterns of distribution among species whose ecological abilities are predicted to be largely equivalent $[19,29]$. In spatial niche models, in contrast, the dispersal limitation acts in conjunction with species specific environmental conditions to determine the distribution of species [31,32]. Chave et al. [19] found that dispersal limitation plays an important role in species distribution for both neutral and niche models.

Since repeatability is an important aspect of community assembly and immigration and dispersal limitation play significant roles in community composition, we investigate in detail the effects of immigration rate and strength of dispersal limitation on repeatability of community composition among replicate communities in both neutral and niche theories using an individual-based spatially explicit model. The neutral model is akin to Gravel et al. [33], while the environment is heterogeneity which remains constant in all simulations and each species has an optimal environmental condition in niche model. The immigrants come from a common regional species pool. The regional species pool is important for community restoration [34] and the first step of ecological restoration is the re-establishment of plant communities at degraded sites through immigration from the regional species pool [34-36]. We examined three different kinds of dispersal limitation: four nearest-neighbors dispersal, the Gaussian dispersal kernel and global dispersal. In order to investigate the repeatability of community composition at the species level (species present or absence) and at the individual level (the abundance of each species), we use the Jaccard and Bray-Curtis similarity indexes [37-39], respectively.

We examine the repeatability among neutral replicate communities and niche replicate communities separately. As higher immigration rates lead to a high probability that species found at the regional scale will also be found in the local community $[8,40]$, repeatability among neutral replicate communities will increase with immigration. In contrast, niche models often rely on deterministic processes and species adapted to the environmental conditions can exist in closed communities. Our first prediction is that, as immigration rate increases, the repeatability among neutral replicate communities will increase while the repeatability among niche replicate communities will decrease. However, much higher immigration should increase the repeatability in both cases because of the high number of individuals coming from the same pool. In our models immigration and dispersal have very different effects. Immigration brings individuals from the regional pool to the local community, while dispersal is the movement of individuals within a local community. Our second prediction is that repeatability among replicate communities will increase as dispersal limitation decreases in both neutral and niche models. It is simply because long dispersal distances leads to homogeneous species distributions in neutral models and species are more likely to find their favorite habitat in niche models. We also investigate the effect of immigration rates and dispersal limitation on repeatability between neutral and niche replicate communities. This is necessary because we can control the immigration or dispersal distance to recover the community from damage, even if we have no idea about the mechanisms maintaining species diversity. In addition, at small and intermediate spatial scales, it is more challenging to distinguish the effects of niche versus neutral processes on community composition $[41,42]$.

\section{Results}

\section{The influence of immigration rate}

The models differ substantially in repeatability. Fig. 1 illustrates the effect of different immigration rates on community repeatability under niche and neutral model. We found that repeatability is average among replicate communities by paired comparison. In the niche model, repeatability first decreases to a critical point, after which it increases (Fig. 1A, 1G). However, for Jaccard similarity, the lowest point appears at $m=10^{-3}$, whereas it occurs at $m=10^{-2.5}$ for Bray-Curtis similarity. With very low and very high immigration rates, Jaccard similarity is 1.0 (Fig. 1A), while Bray-Curtis similarity remains nearly constant at low immigration rates (Fig. 1C). With very low and very high immigration rates, the Jaccard similarity of neutral communities is nearly constant (Fig. 1B). The lowest and the highest points happen at $m=10^{-5}$ and $m=0.1$, respectively. Between these two extremes, Jaccard similarity obviously increases with immigration rate. However, the Bray-Curtis similarity first remains constant and then increases as immigration rate increases (Fig. 1D). The break point appears at $m=10^{-5}$.

The repeatability between neutral and niche communities are affected by immigration rates (Fig. 2). A high immigration rate is needed to generate high repeatability between neutral and niche communities. The highest Bray-Curtis similarity is less than 1.0, while the highest Jaccard similarity is 1.0. For a given immigration rate and community type, increasing the immigration of another community type can increase the repeatability.

The rank-abundance curves become flat as immigration rate increases in both neutral and niche communities (Fig. 3). For a given immigration rate except $m=1$, the relative species abundance curve of niche communities is steeper than for neutral communities. When $m=1$, the rank-abundance curves are similar in flat both at niche and neutral model.

\section{The influence of dispersal limitation}

The effect of dispersal limitation on community repeatability varies between niche and neutral models and the repeatability is averaged among replicate communities by paired comparison in each model (Fig. 4). The repeatability of niche communities increases as dispersal distance increases (Fig. 4A, 4C). Jaccard similarity approaches 1.0 quickly and then remains constant, whilst the Bray-Curtis similarity first increases fast and then increase slow. For neutral communities we find a negative relationship between repeatability and dispersal distance (Fig. 4B, 4D).

The influence of dispersal limitation on repeatability between neutral and niche communities is shown in Fig. 5. When the dispersal distance is large in the niche model and is small in neutral model, the repeatability between them will be large. For a given dispersal distance in the niche model, increasing the dispersal distance in neutral communities decrease repeatability. In contrast, for a given dispersal distance in the neutral model, there is positive correlation between dispersal distance in niche community and repeatability. The highest Jaccard similarity between niche and neutral communities is 1.0, while the highest Bray-Curtis similarity is less than 0.7 .

The effect of dispersal limitation on relative abundance distributions differs between niche and neutral model (Fig. 6). In the niche model, rank- abundance curves become more flat as dispersal distance increases (Fig. 6A), while they become steeper as dispersal distance increases in neutral communities (Fig. 6B). 

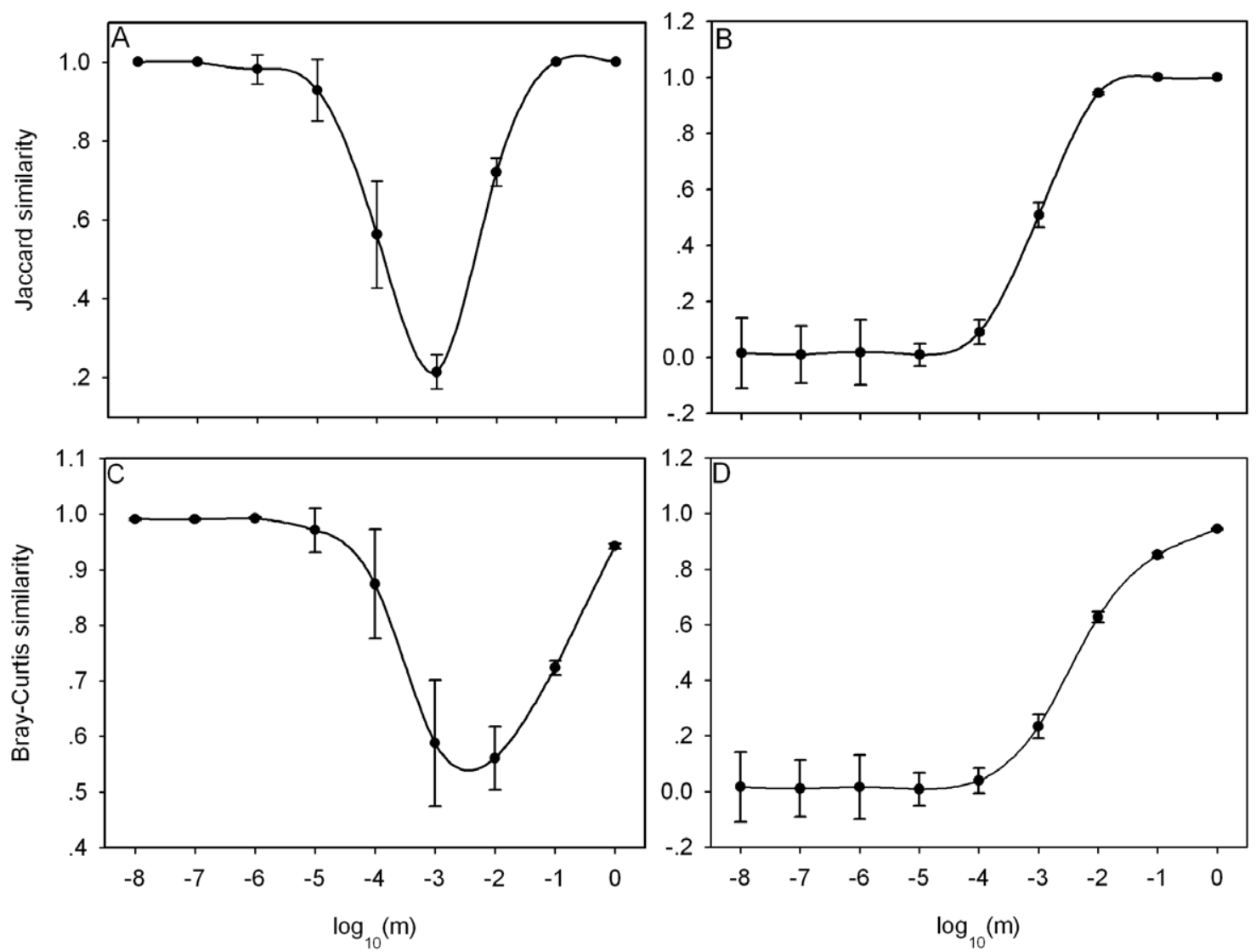

Figure 1. The effect of immigration rates on repeatability of community composition in the different models. A logarithmic scale is used for $m$. Error bars show the standard deviation. Data points are the mean values for 20 replicates. doi:10.1371/journal.pone.0046164.g001

\section{Discussion}

We investigated the influence of immigration rates and dispersal limitation on repeatability in neutral and niche communities separately and between neutral and niche communities together. However, we do find that repeatability differs significantly under niche and neutral models.
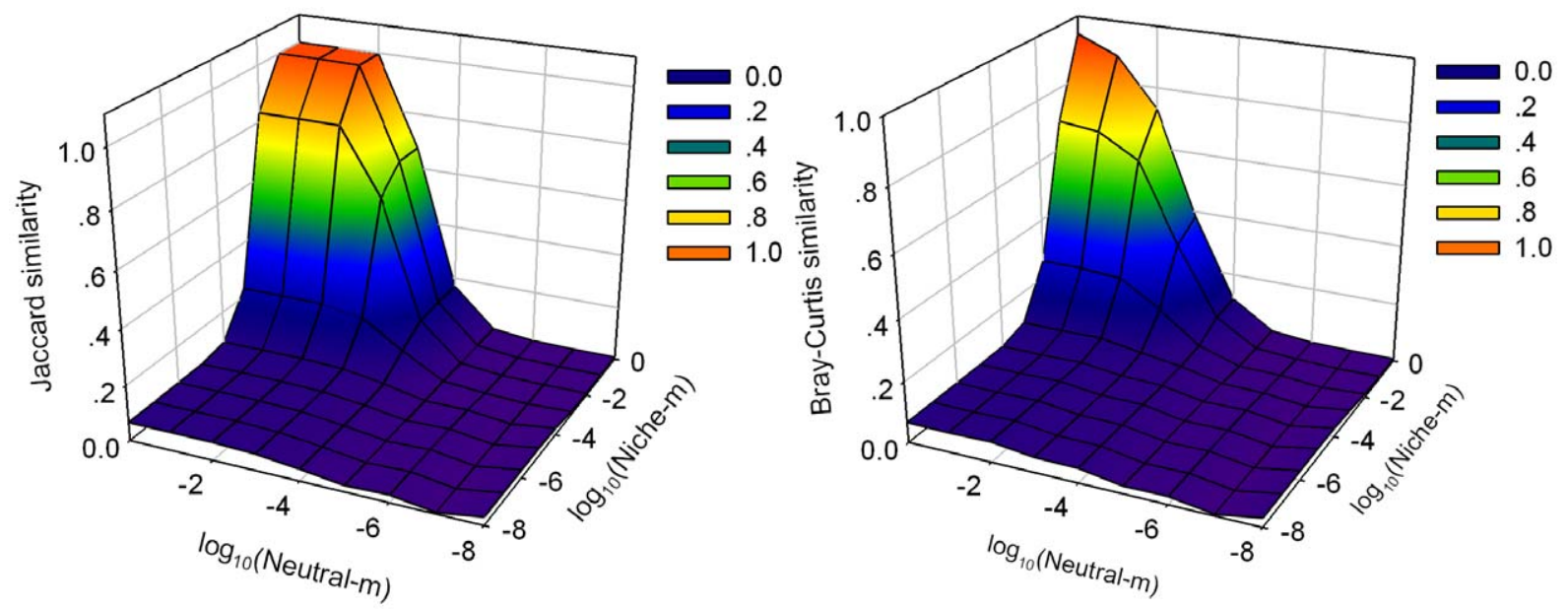

Figure 2. The repeatability between niche and neutral communities under different immigration rates. Neutral- $m$ indicates the immigration of neutral communities and niche- $m$ represents the immigration of niche communities, both on a logarithmic scale. The data points are means for 400 replications.

doi:10.1371/journal.pone.0046164.g002 


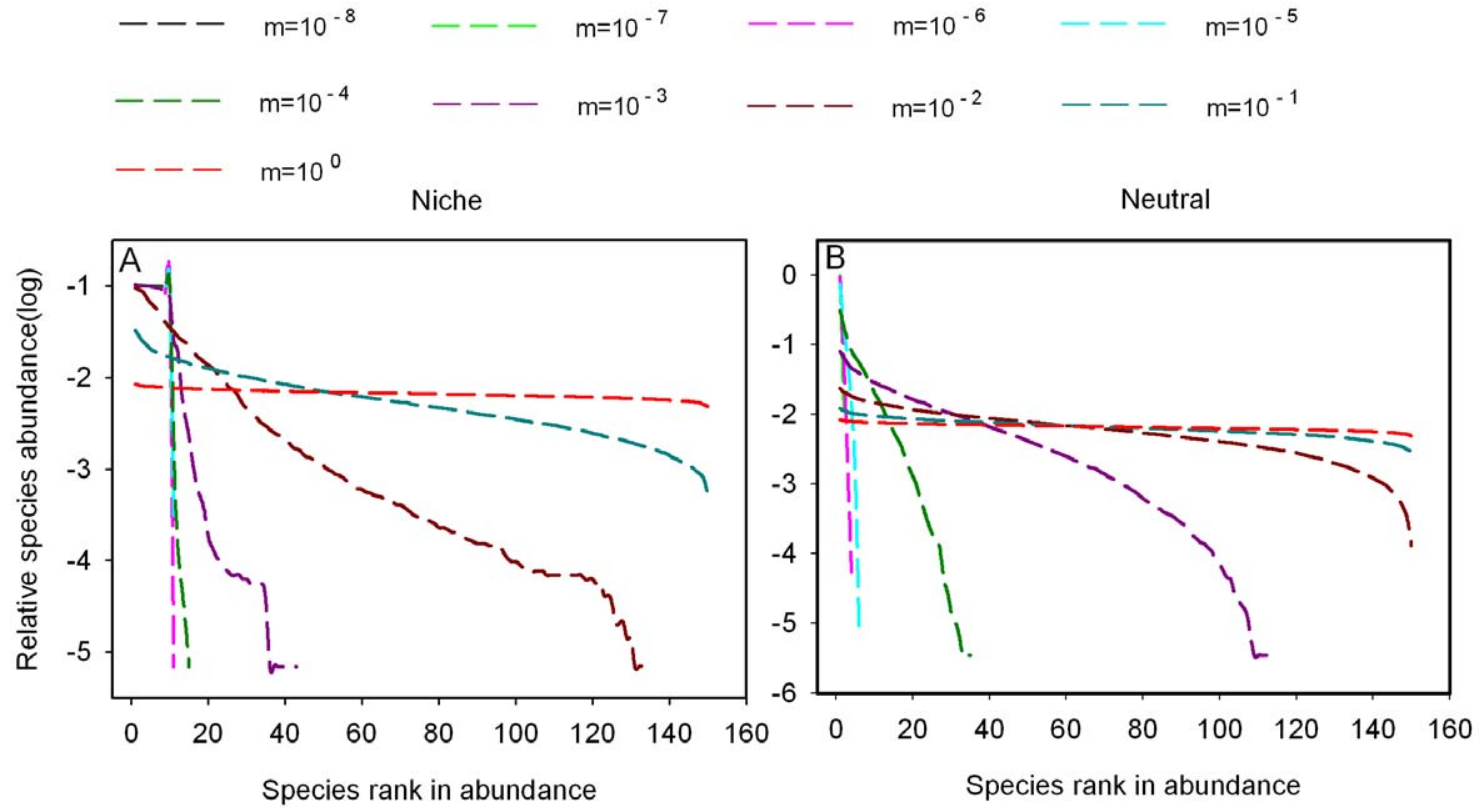

Figure 3. The influence of immigration rates on the equilibrium relative species abundance curves for the different models. Notice that the curves for $\mathrm{m}=10^{-8}$ and $10^{-7}$ overlap. doi:10.1371/journal.pone.0046164.g003
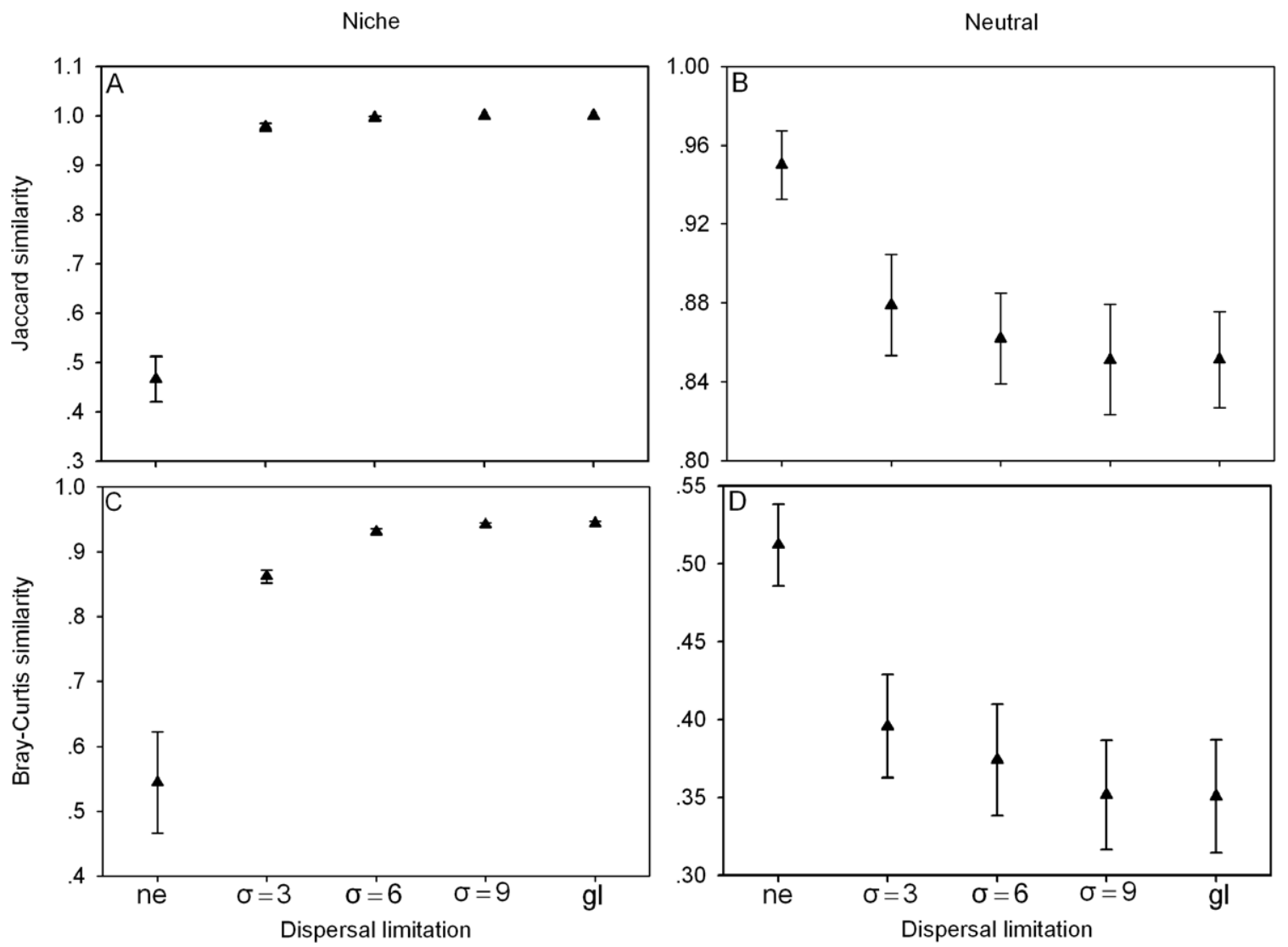

Figure 4. The effect of dispersal limitation on the equilibrium repeatability in the different models. Error bars show standard deviations. $n e$ denotes the four nearest neighbor dispersal, $g /$ denotes global dispersal, and $\sigma$ is the mean dispersal distance in the Gaussian dispersal kernel. For all runs $m=0.005$. The data points are the means for 20 replicates.

doi:10.1371/journal.pone.0046164.g004 

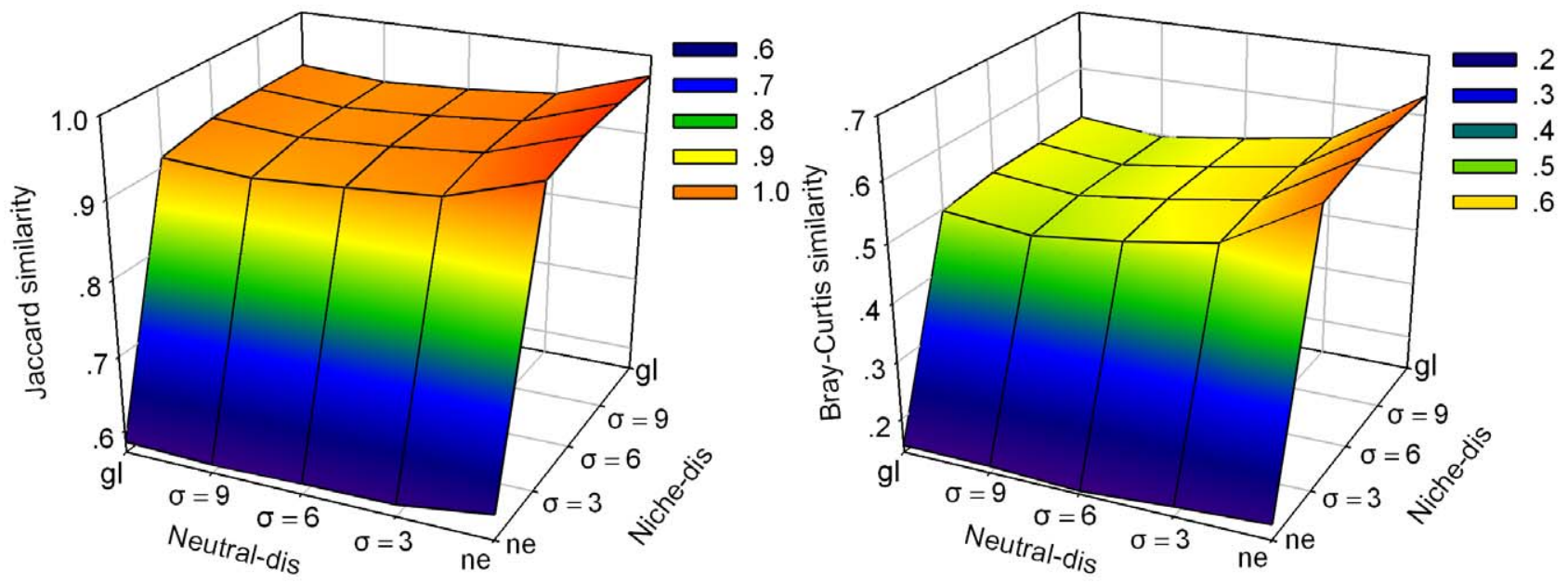

Figure 5. The equilibrium repeatability between niche and neutral communities under different dispersal limitations. Neutral-dis indicates the dispersal distance of neutral communities and niche-dis represents the dispersal distance of niche communities. The default parameters are the same as in Fig. 4.

doi:10.1371/journal.pone.0046164.g005

\section{For immigration rate}

Our results reveal that the neutral theory can produce high repeatability, provided that immigration rate from regional species pool is high. According to the neutral theory, differences in relative abundances are primarily explained by stochastic processes [8]. In addition, species diversity is maintained by the balance between extinction and immigration at the local community scale. In the case of high immigration rates, the local community contains a large number of immigrants and does not differ much in community composition from the regional pool. In contrast, when immigration is restricted, stochastic processes have a strong influence on community composition and repeatability is small. Increasing immigration rates decreases the rate of divergence in diversity among communities [16], so repeatability increases. However, the Jaccard index focuses on species present or not while the Bray-Curtis focuses on relative abundance level, so the Jaccard similarity reaches 1.0 when species composition in two local communities are the same but the Bray-Curtis similarity can hardly reach such high value (Fig. 1B, D).
In niche models, with low immigration rates, some species which can adapt the environmental condition could be maintained in all replicate communities and, as species diversity is mainly influenced by deterministic factors [43,44], so repeatability can be quite high (Fig. 1A, 1G). Increasing immigration leads to an increase in the number of individuals from the regional species pool [45-49]. However, at interdediate migration rate, the individuals coming from regional species pool are limited and the migrants as inferior competitors can be maintained in the communities. According to Tilman [50], propagules must survive long enough to become reproductively successful adults while using the resources left unconsumed by established species. These inferior competitors have difficulties to establish themselves successfully. Added to stochasticity, this leads to lower repeatability of community composition. At much higher immigration rates, although the competitive exclusion is more intense, the high number of immigrants from the regional pool compensates for competitive exclusion and the abundances of all species are distributed evenly in all replicate communities, so repeatability increases.

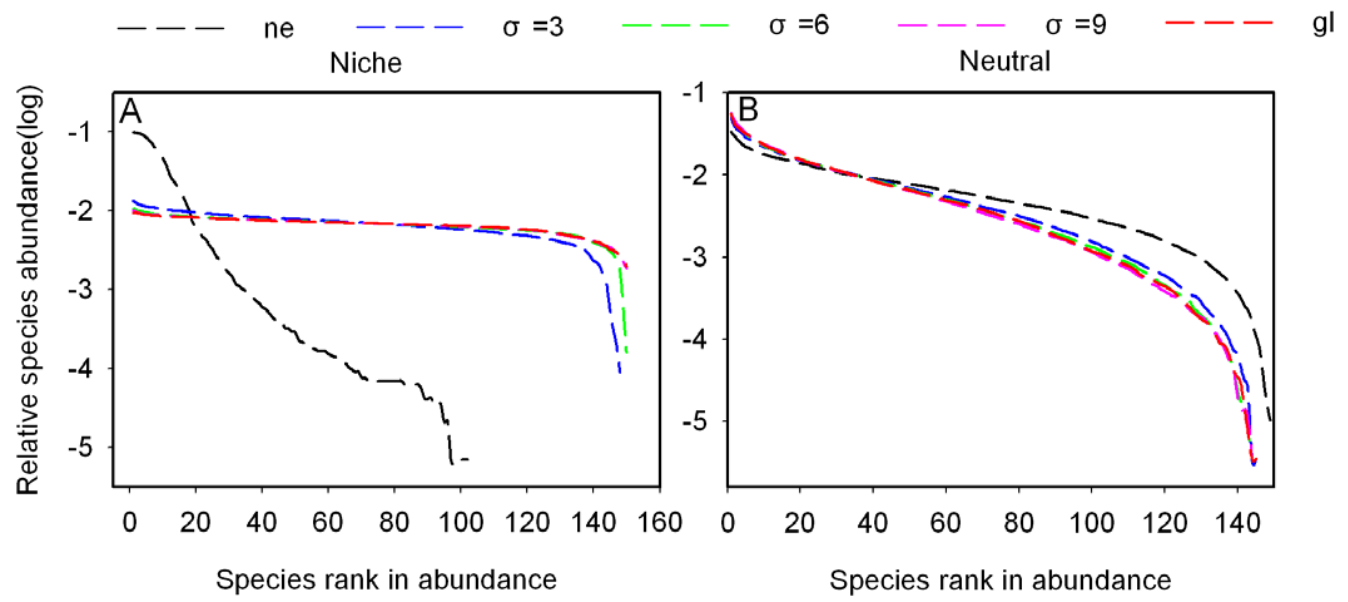

Figure 6. The influence of dispersal limitation on equilibrium relative species abundance curves for the different models. The default parameters are the same as in Fig. 4. doi:10.1371/journal.pone.0046164.g006 
High repeatability is possible between neutral and niche communities when high immigration rates are introduced (Fig. 2). The reason is that high immigration rates increase the probability that a species will appear in both types of communities and abundance of species are more evenly (see Fig. 3). Nevertheless, the highest Bray-Curtis similarity is still lower than the highest Jaccard similarity, because Bray-Curtis index is most sensitive to differences in the relative species abundance [38,39] and some specific species dominate the niche communities even with higher immigration rates. The species adapted to the environmental condition are superior competitiors and can outcompete immigrants, so the relative abundance curves are steeper in niche model than neutral model in which all species have the same competitive ability for a given immigration rates (Fig. 3). Individual death is random in both models, when $m=1$ all dead individuals are replaced by immigrants from the regional species pool, so rankabundance distribution are similar in both models.

\section{For dispersal limitation}

In neutral models, patterns of species distributions are strongly determined by dispersal limitation [19]. In our neutral model, repeatability of community composition decreases as dispersal distance increases, contrasted with the prediction. Under local dispersal, propagules land on sites near their parents [51] and local competition enhances species richness [52], while the propagules could land on all sites of the community under global dispersal and stochastic competitive exclusion increases, which results in the decrease of repeatability (Fig. 4B, D). Interspecific competition will be decreased by the life-history niche difference, because, on average, only those propagules which adapt the environmental condition can land on the empty sites successfully. In our niche model, species with high dispersal have high probability to occupy the favorable empty sites, and then they can escape stochastic extinction and the number of species increases (Fig. 6A), leading to an increase in repeatability (Fig. 4A, 4C).

The repeatability between niche and neutral communities is high only if the dispersal distance of the species in the niche model is large and if it is small in the neutral model (Fig. 5). This may be because increasing dispersal distance results in increasing species richness and reducing the difference among species relative abundance in niche model (see Fig. 6A). On the contrary, species richness decreases and difference among species relative abundance increases as dispersal distance increases in neutral model (see Fig. 6B). These results are consistent with Chave et al. [19], who found that species richness was higher under local dispersal than under longer-distance dispersal.

In this study, we show that repeatability of community composition correlates not only with niche and neutral model but also with immigration rates and dispersal limitation. Understanding how immigration and dispersal limitation affect repeatability of community composition could contribute to the restoration ecology.

\section{Methods}

\section{General framework}

To simulate the repeatability of local communities, we implement an individual-based spatially explicit model. The local community is defined as a two-dimensional lattice. It is divided in $L \times L$ sites and assumed to be a torus. Simulations start with $M$ species and the abundance of each species is $(L \times L) / M$. All individuals are randomly placed on the landscape and each site can be inhabited by a single individual. Our model follows the zero-sum dynamics as neutral theory [8]. At each time step, each individual died with probability $d$, and the empty sites will be occupy either by the immigrants from regional species pool with probability $m$, or by individuals from the same local community.

\section{Neutral model}

In neutral model, all individuals have the same demographic attributes. The fecundity of all species is infinite which means each individual prepares to occupy the site if it is necessary [19]. A dead individual is replaced by a new individual with probability $R_{i}$ [33]:

$$
R_{i}=(1-m) \frac{\sum_{r=1}^{\mathrm{n}} N_{i, r} K(r)}{\sum_{j=1}^{S} \sum_{r=1}^{\mathrm{n}} N_{j, r} K(r)}+m\left(P_{i}\right),
$$

where $r$ is the distance between two sites and $\sigma$ the mean dispersal distance. $\mathcal{N}_{i, r}$ is the group of individuals of species $i$ within some distance $r$ from the recruited site. Parameter $m$ is the probability that a recruit is an immigrant coming from the regional species pool. $K(r)$ is the dispersal kernel. $P_{i}$ is the relative abundance of species $i$ in the regional species pool. The first term is the contribution of the local population of species $i$ to the propagule supply. The second term is the contribution of the regional species pool of species $i$ to the propagule supply.

We examined three different kinds of dispersal limitation: global dispersal, four nearest-neighbors dispersal and the Gaussian dispersal kernel [19] that takes the form:

$$
K(r)=\frac{r}{\sigma^{2}} \exp \left(-\frac{r^{2}}{2 \sigma^{2}}\right) .
$$

\section{Niche model}

In real communities, species differ on many aspects. In niche model, we assume species differ in environmental niche optima and the landscape of communities is a simple monotonic environmental gradient which is unaltered in the simulation process. We use monotonic gradients in the model for the sake of simplicity; such gradients may be observed in nature at small spatial scales, or in particular habitats (e.g. vernal pools and steep elevation gradients). Following Schwilk and Ackerly [53] the value of the environmental factor $E$ is a linear function of location on the landscape:

$$
E_{x}=E_{\min }+\left(\frac{x}{X}\right) E_{\text {range }}
$$

We equally divide the landscape into $X$ small sections and number sections from 0 to $X-1 . E_{x}$ is the $E$ value of section $x, E_{\text {min }}$ and $E_{\text {range }}$ are the minimal value and the range of $E$, respectively.

When there is niche differentiation, the survival probability of species $i$ at location $x, \lambda_{i, x}$, is associated with environmental factor $E$ of location $x$, species optimal environmental condition $\mu_{i}$ and niche breadth $w$ [33], as follows:

$$
\lambda_{i, x}=\exp \left[-\frac{\left(E_{x}-\mu_{i}\right)^{2}}{2 w^{2}}\right]
$$


The niche breadth (w) of each species is identical. We label the species from 1 to $M$ and the value of the optimal environmental condition of species $i$ is [53]:

$$
\mu_{i}=\frac{(i-1)}{M} E_{\text {range }}+\frac{1}{2 M}
$$

The probability that a recruit will be of species $i$ in a given environment $x$ is:

$$
R_{i, x}=(1-m) \frac{\lambda_{i, x}}{\sum_{j=1}^{s} \lambda_{j, x}}+m\left(P_{i}\right)
$$

where $s$ is the number of species in given dispersal distance.

\section{Similarity indexes}

Repeatability is measured by both the Jaccard similarity index and the Bray-Curtis similarity index. The Jaccard similarity index is based on presence-absence data and is defined as the number of species shared in both communities divided by the number of species in the two communities [37]. It is sensitive to species richness but not to relative species abundance. However, the BrayCurtis index is much more sensitive to differences in relative abundance, although it is also affected by species richness $[38,39,54]$. Both indices have been widely used in community studies and shown to provide robust estimates of the differences among community structures [37].

\section{Simulations performed}

We repeat the simulations for each community 20 times. For a given community type (either neutral community or niche community) and parameter setting, we examine the repeatability

\section{References}

1. Poulin R, Valtonen ET (2002) The predictability of helminth community structure in space: a comparison of fish populations from adjacent lakes. Int J Parasitol 32: 1235-1243.

2. Keith AR, Bailey JK, Whitham TG (2010) A genetic basis to community repeatability and stability. Ecology 91: 3398-3406.

3. Sharik TL, Ford RH, Davis ML (1989) Repeatability of invasion of eastern white pine on dry sites in northern lower Michigan. Am Midl Nat 122: 133-141.

4. Gonzàlez MT, Poulin R (2005) Spatial and temporal predictability of the parasite community structure of a benthic marine fish along its distributional range. Int J Parasitol 35: 1369-1377.

5. Lawton JH, Gaston KJ (1989) Temporal patterns in the herbivorous insects of bracken: a test of community predictability. J Anim Ecol 58: 1021-1034.

6. Palmer MA, Ambrose RF, Poff NL (1997) Ecological theory and community restoration ecology. Restor Ecol 5: 291-300.

7. Ruokolainen L, Ranta E, Kaitala V, Fowler MS (2009) When can we distinguish between neutral and non-neutral processes in community dynamics under ecological drift? Ecol Lett 12: 1-11.

8. Hubbell SP (2001) The unified neutral theory of biodiversity and biogeography. Princeton: Princeton Univ. Press.

9. Bell G (2000) The distribution of abundance in neutral communities. Am Nat 155: 606-617.

10. Tilman D (1994) Competition and biodiversity in spatially structured habitats. Ecology 75: 2-16.

11. Tilman D, Pacala S (1993) The maintenance or species richness in plant communities. In: Ricklefs RE, Schluter D, editors. Species Diversity in Ecological Communities. Chicago: University of Chicago Press. pp. 13-25.

12. Parrish JAD, Bazzaz FA (1976) Underground niche separation in successional plants. Ecology 57: 1281-1288.

13. Tilman D (1982) Resource competition and community structure, Monographs in population biology. Princeton: Princeton Univ. Press.

14. Chesson P (2000) Mechanisms of maintenance of species diversity. Annu Rev Ecol Syst 31: 343-366.

15. Volkov I, Banavar JR, Maritan A, Hubbell SP (2004) The stability of forest biodiversity. Nature 427: 696 . among these replicate communities and then take the average. To investigate the repeatability between neutral and niche communities, and for a given parameter setting, we examine the similarity between 20 neutral replicate communities and 20 niche replicate communities by paired comparison. There are nine immigration rate values with $m=\left\{10^{-8}, 10^{-7}, 10^{-6}, 10^{-5}, 10^{-4}, 10^{-3}, 10^{-2}\right.$, $\left.10^{-1}, 10^{0}\right\}$ and several dispersal kernels: nearest dispersal, global dispersal and Gaussian dispersal with mean dispersal distance $\sigma=\{3,6,9\}$. For a given simulation, mean dispersal distance of all individuals is equal. Simulations were performed on lattices with $L=120$ and an initial species number of $M=40$. The mortality rate is $d=0.1$. In niche model, the landscape is equally divided into $X=10$ sections and the $E_{\text {min }}=0$ and $E_{\text {rang }}=1.0$. Both the neutral and niche models share the same regional species pool which contains 150 species and the distribution of species relative abundances is a stationary uniform. For niche model, the optimal environmental condition of each species in regional pool is also assigned by equation 5 . We perform each simulation for $10^{6}$ time steps and present the results in the form of a mean and/or a standard deviation. For the Jaccard similarity index and BrayCurtis similarity index, we use the fossil package [55] implemented in the $\mathrm{R}$ version 2.13.1 [56].

\section{Acknowledgments}

We thank Sa Xiao for help with model design, Renyi Zhang, Zhengwei Ren, Yingbo Yang and Rui Hou for helpful comments on the manuscript. We also thank the editor and two anonymous reviewers for improving the quality of the manuscript.

\section{Author Contributions}

Conceived and designed the experiments: DA GW. Performed the experiments: DA CC. Analyzed the data: DA PDP. Contributed reagents/materials/analysis tools: DA CG. Wrote the paper: DA PDP GW.

16. Maurer BA, McGill BJ (2004) Neutral and non-neutral macroecology. Basic Appl Ecol 5: 413-422.

17. Hubbell SP (1997) A unified theory of biogeography and relative species abundance and its application to tropical rain forests and coral reefs. Coral Reefs 16(suppl.): S9-S21.

18. Loreau M, Mouquet N (1999) Immigration and the maintenance of local species diversity. Am Nat 154: 427-440.

19. Chave J, Muller-Landau HC, Levin SA (2002) Comparing classical community models: theoretical consequences for patterns of diversity. Am Nat 159: 1-23.

20. Chase JM (2003) Community assembly: when should history matter? Oecologia 136: 489-498.

21. Chase JM (2007) Drought mediates the importance of stochastic community assembly. Proc Natl Acad Sci U S A 104: 17430-17434.

22. Armstrong RA, McGehee R (1980) Competitive exclusion. Am Nat 115: 151170.

23. Levin SA (1970) Community equilibria and stability, and an extension of the competitive exclusion principle. Am Nat 104: 413-423.

24. MacArthur RH, Levins R (1964) Competition, habitat selection, and character displacement in a patchy environment. Proc Natl Acad Sci U S A 51: 12071210

25. Soininen J, McDonald R, Hillebrand H (2007) The distance decay of similarity in ecological communities. Ecography 30: 3-12.

26. Shmida A, Wilson MV (1985) Biological determinants of dpecies diversity. J Biogeogr 12: 1-20.

27. Brown JH, Kodric-Brown A (1977) Turnover rates in insular biogeography: effect of immigration on extinction. Ecology 58: 445-449.

28. Hubbell SP (1979) Tree dispersion, abundance, and diversity in a tropical dry forest. Science 203: 1299-1309.

29. Chave J, Leigh EG (2002) A spatially explicit neutral model of $\beta$-diversity in tropical forests. Theor Popul Biol 62: 153-168.

30. Zhou SR, Zhang DY (2008) A nearly neutral model of biodiversity. Ecology 89: 248-258.

31. Gilbert B, Lechowicz MJ (2004) Neutrality, niches, and dispersal in a temperate forest understory. Proc Natl Acad Sci U S A 101: 7651-7656. 
32. Snyder RE, Chesson P (2003) Local dispersal can facilitate coexistence in the presence of permanent spatial heterogeneity. Ecol Lett 6: 301-309.

33. Gravel D, Canham CD, Beaudet M, Messier C (2006) Reconciling niche and neutrality: the continuum hypothesis. Ecol Lett 9: 399-409.

34. Zobel M, van der Maarel E, Dupré C (1998) Species pool: the concept, its determination and significance for community restoration. Appl Veg Sci 1: 5566.

35. Rui-Jaen MC, Aide TM (2005) Restoration success: how is it being measured. Restor Ecol 13: 569-577.

36. Brudvig LA, Mabry CM (2008) Trait-based filtering of the regional species pool to guide understory plant reintroductions in Midwestern Oak Savannas, U.S.A. Restor Ecol 16: 290-304.

37. Irigoien X, Chust G, Fernandes JA, Albaina A, Zarauz L (2011) Factors determining the distribution and betadiversity of mesozooplankton species in shelf and coastal waters of the Bay of Biscay. J Plankton Res 0: 1-11.

38. Davies KF, Melbourne BA, Margules CR (2001) Effects of within-and between patch processes on community dynamics in a fragmentation experiment. Ecology 82: 1830-1846.

39. Bray JR, Curtis JT (1957) An ordination of the upland forest communities of southern Wisconsin. Ecol Monogr 27: 325-349.

40. Chase JM, Kraft NJB, Smith KG, Vellend M, Inouye BD (2011) Using null models to disentangle variation in community dissimilarity from variation in $\alpha$ diversity. Ecosphere 2: Article 24.

41. Bell G, Lechowicz MJ, Waterway MJ (2001) The precision of adaptation in forest plants. In: Silvertown J, Antonovics J, editors. Integrating ecology and evolution in a spatial context. London: Blackwell Scientific. pp.117-135.

42. Duivenvoorden JF, Svenning JC, Wright SJ (2002) Beta diversity in tropical forests. Science 295: 636-637.

43. Mouquet N, Loreau M (2003) Community patterns in source-sink metacommunities. Am Nat 162: 544-557.
44. Mouquet N, Miller TE, Daufresne T, Kneitel JM (2006) Consequences of varying regional heterogeneity in source-sink metacommunities. Oikos 113: 481-488.

45. Cadotte MW (2006) Metacommunity influences on community richness at multiple spatial scales: a microcosm experiment. Ecology 87: 1008-1016.

46. McKane AJ, Alonso D, Solé RV (2004) Analytic solution of Hubbell's model of local community dynamics. Theor Popul Biol 65: 67-73.

47. Leibold MA, Norberg J (2004) Biodiversity in metacommunities: plankton as complex adaptive systems? Limnol Oceanogr 49: 1278-1289.

48. Leibold MA, Holyoak M, Mouquet N, Amarasekare P, Chase JM, et al. (2004) The metacommunity concept: a framework for multi-scale community ecology. Ecol Lett 7: 601-613.

49. Shurin JB (2000) Dispersal limitation, invasion resistance, and the structure of pond zooplankton communities. Ecology 81: 3074-3086.

50. Tilman D (2004) Niche tradeoffs, neutrality, and community structure: a stochastic theory of resource competition, invasion, and community assembly. Proc Natl Acad Sci U S A 101: 10854-10861.

51. Holyoak M, Loreau M (2006) Reconciling empirical ecology with neutral community models. Ecology 87: 1370-1377.

52. Laird RA, Schamp BS (2008) Does local competition increase the coexistence of species in intransitive networks? Ecology 89: 237-247.

53. Schwilk DW, Ackerly DD (2005) Limiting similarity and functional diversity along environmental gradients. Ecol Lett 8: 272-281.

54. Faith DP, Minchin PR, Belbin L (1987) Compositional dissimilarity as a robust measure of ecological distance. Vegetation 69: 57-68.

55. Vavrek MJ (2011) fossil: palaeoecological and palaeogeographical analysis tools. Palaeontol Electronica 14:1T-16p.

56. R Development Core Team (2009) R: a language and environment for statical computing. Vienna: R Foundation for Statical Computing. 